\title{
Iterative Combinatorial Auctions
}

\section{Citation}

Parkes, David C. 2006. Iterative combinatorial auctions. In Combinatorial Auctions, ed. P. Cramton, Y. Shoham, and R. Steinberg, 41-78. Cambridge, Mass: MIT Press.

\section{Published Version}

http://mitpress.mit.edu/main/home/default.asp

\section{Permanent link}

http://nrs.harvard.edu/urn-3:HUL.InstRepos:4000814

\section{Terms of Use}

This article was downloaded from Harvard University's DASH repository, and is made available under the terms and conditions applicable to Other Posted Material, as set forth at http:// nrs.harvard.edu/urn-3:HUL.InstRepos:dash.current.terms-of-use\#LAA

\section{Share Your Story}

The Harvard community has made this article openly available.

Please share how this access benefits you. Submit a story.

Accessibility 


\title{
Chapter 2: Iterative Combinatorial Auctions
}

\author{
David C. Parkes
}

\section{Introduction}

Combinatorial auctions allow bidders to express complex valuations on bundles of items, and have been proposed in settings as diverse as the allocation of floor space in a new condominium building to individual units (Wired 2000) and the allocation of take-off and landing slots at airports (Smith, Forward). Many applications are described in Part V of this book.

The promise of combinatorial auctions (CAs) is that they can allow bidders to better express their private information about preferences for different outcomes and thus enhance competition and market efficiency. Much effort has been spent on developing algorithms for the hard problem of winner determination once bids have been received (Sandholm, Chapter 14). Yet, preference elicitation has emerged as perhaps the key bottleneck in the real-world deployment of combinatorial auctions. Advanced clearing algorithms are worthless if one cannot simplify the bidding problem facing bidders.

Preference elicitation is a problem both because of the communication cost of sending bids to the auction and also because of the cost on bidders to determine their valuations for different bundles. The problem of communication complexity can be addressed through the design of careful bidding languages, that provide expressive but concise bids (Nisan Chapter 9). Non-computational approaches can also be useful, such as defining the good and bundle space in the right way in the first place (Pekeč and Rothkopf Chapter 16).

However, even well-designed sealed-bid auctions cannot address the problem of hard valuation problems because they preclude the use of feedback and price 
discovery to focus bidder attention. There are an exponential number of bundles to value in CAs. Moreover, the problem of valuing even a single bundle can be difficult in many applications of CA technology. For instance, in the airport landing slot scenario (see Ball, Donohue and Hoffman Chapter 20) we should imagine that airlines are solving local scheduling, marketing, and revenue-management problems to determine their values for different combinations of slots.

Iterative combinatorial auctions are designed to address the problem of costly preference elicitation that arises due to hard valuation problems. An iterative CA allows bidders to submit multiple bids during an auction and provides information feedback to support adaptive and focused elicitation. For example, an ascending price auction maintains ask prices and allows bidders to revise bids as prices are discovered. Significantly, it is often possible to determine an efficient allocation without bidders reporting, or even determining, exact values for all bundles. In contrast, any efficient sealed-bid auction requires bidders to report and determine their value for all feasible bundles of goods.

This ability to mitigate the preference elicitation problem is a central concern in iterative CA design. But there are also a number of less tangible yet still important benefits:

- Iterative CAs can help to distribute the computation in an auction across bidders through the interactive involvement of bidders in guiding the dynamics of the auction. Some formal models show the equivalence between iterative CAs and decentralized optimization algorithms (Parkes and Ungar 2000a, de Vries, Schummer, and Vohra 2003). Iterative CAs can address concerns about privacy because bidders only need to reveal partial and indirect information about their valuations. ${ }^{1}$

- Transparency is another practical concern in CAs. In the high-stakes world of wireless spectrum auctions, the Federal Communications Commission 
(FCC) has been especially keen to ensure that bidders can verify and validate the outcome of an auction. Although mathematically elegant, the VCG outcome can be difficult to explain to bidders, and validation requires the disclosure and verification of many bids, both losing and winning. Thus, even as readily describable implementations of sealed-bid auctions, iterative CAs can offer some appeal (Ausubel and Milgrom 2002).

- The dynamic exchange of value information between bidders, that is enabled within iterative CAs, is known to enhance revenue and efficiency in single item auctions with correlated values (Milgrom and Weber 1982). Although little is known about the design of iterative CAs for correlated value problems, one should expect iterative CAs to retain this benefit over their sealed-bid counterparts. Certainly, correlated value settings exist: consider the wireless spectrum auctions in which valuations are in part driven by underlying population demographics and shared technological realities.

Yet, even with all these potential advantages iterative CAs offer new opportunities to bidders for manipulation. The biggest challenge in iterative CA design is to support incremental and focused bidding without allowing new strategic behavior to compromise the economic goals of efficiency or optimality. For instance, one useful design paradigm seeks to implement auctions in which straightforward bidding (truthful demand revelation in response to prices) is an ex post equilibrium. This equilibrium is invariant to the private information of bidders, so that straightforward bidding is a best response whatever the valuations of other bidders.

Steps can also be taken to minimize opportunities for signaling through careful control of the information that can be shared between bidders during an auction. Finally, the benefits of iterative auctions disappear when bidders choose to strategically delay bidding activity until the last rounds of an auction. Activity 
rules (Milgrom 2000) can be used to address this stalling and promote meaningful bidding during the early rounds of an auction.

The existing literature on iterative CAs largely focuses on the design of efficient auctions. Indeed, there are no known optimal (i.e. revenue-maximizing) general-purpose CAs, iterative or otherwise. As such, the canonical VCG mechanism (see Chapter 1) has guided the design of many iterative auctions. ${ }^{2}$ We focus mainly on price-based approaches, in which the auctioneer provides ask prices to coordinate the bidding process. We also consider alternative paradigms, including decentralized protocols, proxied auctions in which a bidding agent elicits preference information and automatically bids using a predetermined procedure, and direct-elicitation approaches.

In outline, Section 2 defines competitive equilibrium (CE) prices for CAs, which may be non-linear and non-anonymous in general. Connections between $\mathrm{CE}$ prices, the core of the coalitional game, and the VCG outcome are explained. Section 3 describes the design space of iterative CAs. Section 4 discusses price-based auctions, providing a survey of existing price-based CAs in the literature and a detailed case study of an efficient ascending price auction. Section 5 considers some alternatives to price-based design. Section 6 closes with a brief discussion of some of the open problems in the design of iterative combinatorial auctions, and draws some connections with the rest of this book.

\section{Preliminaries}

Let $\mathcal{G}=\{1, \ldots, m\}$ denote the set of items, and assume a private values model with $v_{i}(S) \geq 0$ to denote the value of bidder $i \in \mathcal{I}=\{1, \ldots, n\}$ for bundle $S \subseteq \mathcal{G}$. Note that set $\mathcal{I}$ does not include the seller. We assume free-disposal, with $v_{i}(T) \geq v_{i}(S)$ for all $T \supseteq S$, and normalization, with $v_{i}(\emptyset)=0$. Let $\mathcal{V}$ denote the set of bidder valuations. Bidders are assumed to have quasi-linear utility (we also use payoff interchangeably with utility), with utility $u_{i}(S, p)=v_{i}(S)-p$ for bundle $S$ at price $p \geq 0$. This assumes the absence of any budget constraints. 
Further assume that the seller has no intrinsic value for the items.

The efficient combinatorial allocation problem (CAP) solves:

$$
\begin{aligned}
& \max _{S=\left(S_{1}, \ldots, S_{n}\right)} \sum_{i \in \mathcal{I}} v_{i}\left(S_{i}\right) \\
& \text { s.t. } \quad S_{i} \cap S_{j}=\emptyset, \quad \forall i, j
\end{aligned}
$$

Let $S^{*}$ denote the efficient allocation. Also, we write $\operatorname{CAP}(\mathcal{I} \backslash i)$ to denote the combinatorial allocation problem without bidder $i$.

\subsection{Competitive Equilibrium Prices}

We can consider a hierarchical structure for ask prices in CAs:

Linear. Prices $p_{j} \geq 0$, for $j \in \mathcal{G}$, define additive prices on bundles, with

$$
p(S)=\sum_{j \in S} p_{j} .
$$

Non-linear. Prices, $p(S) \geq 0$, for $S \subseteq \mathcal{G}$, allow $p(S) \neq p\left(S_{1}\right)+p\left(S_{2}\right)$, for some $S=S_{1} \cup S_{2}$ and $S_{1} \cap S_{2}=\emptyset$.

Non-linear and Non-anonymous. Prices $p_{i}(S) \geq 0$, allow discriminatory pricing, with $p_{i}(S) \neq p_{i^{\prime}}(S)$ for bidder $i \neq i^{\prime}$, in addition to non-linear prices.

In the following definitions we adopt $p_{i}(S)$ for notational convenience. We intend to allow (but not require) with this notation non-linear and non-anonymous prices. For instance, linear prices $p_{j}$ can be considered to induce prices $p_{i}(S)=\sum_{j \in S} p_{j}$ for bundle $S$ and bidder $i$.

Competitive equilibrium prices extend the concept of Walrasian equilibrium prices to a CA. Let $\pi_{i}(S, p)=v_{i}(S)-p_{i}(S)$ denote bidder $i$ 's payoff from bundle $S$ at prices $p$ and $\Pi_{s}(S, p)=\sum_{i \in \mathcal{I}} p_{i}\left(S_{i}\right)$ denote the seller's revenue from allocation $S$ at prices $p$. 
Definition 1 (Competitive Equilibrium). Prices, $p$, and allocation $S^{*}=\left(S_{1}^{*}, \ldots, S_{n}^{*}\right)$ are in competitive equilibrium $(C E)$ if:

$$
\begin{aligned}
\pi_{i}\left(S_{i}^{*}, p\right) & =\max _{S \subseteq \mathcal{G}}\left[v_{i}(S)-p_{i}(S), 0\right] \quad \forall i \\
\Pi_{s}\left(S^{*}, p\right) & =\max _{S \in \Gamma} \sum_{i \in \mathcal{I}} p_{i}\left(S_{i}\right)
\end{aligned}
$$

where $\Gamma$ denotes the set of all feasible allocations.

A competitive equilibrium $\left(p, S^{*}\right)$ is such that allocation $S^{*}$ maximizes the payoff of every bidder and the seller given prices. Allocation $S^{*}$ is said to be supported by prices $p$ in CE.

Theorem 1. Allocation $S^{*}$ is supported in competitive equilibrium if and only if $S^{*}$ is an efficient allocation.

This welfare theorem follows from a simple linear-programming (LP) duality argument for suitably extended LP formulations of the CAP (Bikhchandani and Ostroy 2002, also Chapter 8). Moreover, CE prices always exist for the CAP. For instance, prices $p_{i}=v_{i}$ trivially satisfy the $\mathrm{CE}$ conditions. The main new element in CAs is that these CE prices must sometimes be non-linear and non-anonymous. Bikhchandani and Ostroy also show an equivalence between the core of the coalitional game and the set of CE prices. All core outcomes can be priced, and all CE prices correspond to core payoffs.

Many iterative CAs are designed to converge to CE prices, and as such it is important to characterize classes of valuations for which linear, and non-linear but anonymous, CE prices exist. We will also see that it is necessary that an efficient CA must determine enough information about bidder valuations to define a set of CE prices, and necessary that a Vickrey auction determines enough information to define a set of universal CE prices.

For the existence of linear CE prices, it is sufficient (and almost necessary) ${ }^{3}$ that valuations satisfy a goods are substitutes property (Kelso and Crawford 1982, Gul 
and Stacchetti 1999). This substitutes condition is defined indirectly, with respect to a demand set:

$$
D_{i}(p)=\left\{S: \pi_{i}(S, p) \geq \max _{T \subseteq \mathcal{G}} \pi_{i}(T, p), \pi_{i}(S, p) \geq 0, S \subseteq \mathcal{G}\right\},
$$

which includes all bundles that maximize a bidder's payoff at the prices.

Definition 2 (Goods are Substitutes). Valuation $v_{i}$ satisfies goods are substitutes if for all linear prices $p, p^{\prime}$ such that $p^{\prime} \geq p$ (component-wise), and all $S \in D_{i}(p)$, there exists $T \in D_{i}\left(p^{\prime}\right)$ such that $\left\{j \in S: p_{j}=p_{j}^{\prime}\right\} \subseteq T$.

The goods are substitutes (or simply substitutes) condition requires that a bidder will continue to demand items that do not change in price as the price on other items increases. Substitutes valuations include unit-demand valuations with $v_{i}(S)=\max _{j \in S}\left\{v_{i j}\right\}$ for all $S$ and value $v_{i j}$ on item $j$ in isolation, but preclude the possibility of items with complementary values (Lehmann, Lehmann, and Nisan 2001).

Conditions for the existence of non-linear but anonymous CE prices are less well-understood, but sufficient conditions presented in Parkes (2001) (Theorem 4.7) include supermodular valuations, single-minded bidders that value a particular bundle, and bidders with safe valuations such that each pair of bundles with positive value to a bidder share at least one item. Consider, for example, a bidder in the FCC spectrum auction that definitely needs lower Manhattan, along with as many of the geographically neighboring licenses as possible.

\subsection{Minimal Competitive Equilibrium Prices}

In fact, many iterative $\mathrm{CAs}$ are designed to converge to minimal $\mathrm{CE}$ prices. This can be useful for two reasons. First, minimal CE prices on bundles in the efficient allocation correspond to VCG payments for a restricted class of valuations. In this case, we say that the CE prices support the VCG payments. Termination with CE prices that support VCG payments brings straightforward bidding into an ex post equilibrium. Second, Ausubel and Milgrom (2000, also Chapter 3) show that 
implementing minimal CE prices (corresponding to buyer-optimal core outcomes) avoids the problems that can occur with the VCG auction when VCG payments are not supported with minimal CE prices.

Definition 3 (Minimal CE Prices). Minimal CE prices minimize the seller's total revenue $\Pi_{s}\left(S^{*}, p\right)$ on the efficient allocation $S^{*}$ across all CE prices. A bidder's payment in the VCG mechanism is always less than or equal to the payment by that bidder at any CE price (Bikhchandani and Ostroy 2002). Thus, minimal CE prices always provide an upper-bound on VCG payments. Moreover, a bidder's VCG payment is equal to the CE price on her efficient bundle in some CE (Parkes and Ungar 2000b).

A characterization in terms of the coalitional value function explains when the VCG can be supported simultaneously to all bidders in the minimal CE. Let $w(L)$ for $L \subseteq \mathcal{I}$ denote the coalitional value for a subset $L$ of bidders, equal to the value of the efficient allocation for $\mathrm{CAP}(L)$. The buyers are substitutes (BAS) condition requires,

$$
w(\mathcal{I})-w(\mathcal{I} \backslash K) \geq \sum_{i \in K}[w(\mathcal{I})-w(\mathcal{I} \backslash i)], \quad \forall K \subset \mathcal{I}
$$

Theorem 2. (Bikhchandani and Ostroy 2002) A buyers are substitutes (BAS) coalitional value function is necessary and sufficient to support the VCG payments in competitive equilibrium.

In particular, the VCG payments are implemented in the minimal CE (or buyer-optimal core) when BAS holds, and buyer-optimal core payoffs are unique exactly when BAS holds.

A number of ascending price CAs can only terminate with minimal CE prices given a slightly stronger condition, that of a buyer-submodular (BSM) coalitional value function:

$$
w(L)-w(L \backslash K) \geq \sum_{i \in K}[w(L)-w(L \backslash i)], \quad \forall K \subset L, \forall L \subseteq \mathcal{I}
$$


Bikhchandani and Ostroy (Chapter 8) refer to BSM as buyers are strong substitutes. Clearly, a BSM coalitional value function also satisfies BAS. But there are cases for which values satisfy BAS but not BSM (see Ausubel and Milgrom 2002, Section 7, for example). Interestingly, substitutes valuations implies BSM and is almost necessary. Roughly, if at least one bidder does not satisfy substitutes then one can construct substitutes valuations for other bidders such that the coalitional value function fails BSM. See Ausubel and Milgrom (Chapter 1) for further discussion. Thus, the same conditions for the existence of a linear price equilibrium are sufficient and almost necessary for the existence of some price equilibrium (although perhaps non-linear and non-anonymous) that supports the Vickrey outcome. ${ }^{4}$

\subsection{Universal Competitive Equilibrium Prices}

Experiments have suggested that BAS can often fail in realistic settings for CAs. ${ }^{5}$ In these cases the VCG payments are not supported in any price equilibrium. We can still design price-based CAs by characterizing a stronger condition on $\mathrm{CE}$ prices that implies enough information to determine VCG payments from these prices. For this, we restrict attention to the universal CE prices (Parkes and Ungar 2002, Mishra and Parkes 2004).

\section{Definition 4 (Universal CE Prices). Prices p are universal Competitive}

Equilibrium (UCE) prices if:

a) Prices p are CE prices.

b) Prices $p_{-i}$ are $C E$ prices for $C A P(\mathcal{I} \backslash i)$, meaning they support some efficient allocation in $C A P(\mathcal{I} \backslash i)$, for all bidders $i$.

where $p_{-i}=\left(p_{1}, \ldots, p_{i-1}, p_{i+1}, \ldots, p_{n}\right)$.

In words, prices are UCE when an efficient allocation for the restricted allocation problem without bidder $i$ is supported with prices $p_{-i}$, for each bidder $i$ removed 
in turn. Thus, UCE prices are CE prices in the main economy and in every marginal economy. Note that UCE prices need not require that the same allocation is supported in every marginal economy. The prices must support some efficient allocation in each marginal economy. ${ }^{6}$ UCE prices always exist, for example $p_{i}=v_{i}$, for all bidders $i$, are UCE prices. Moreover, a universal price equilibrium provides sufficient information about bidder valuations to compute the VCG outcome.

Theorem 3. (Parkes and Ungar 2002) Given a UCE with prices $p_{\mathrm{uce}}$ and an efficient allocation $S^{*}$, the VCG payment to bidder $i$ is computed as:

$$
p_{\mathrm{vcg}, i}=p_{\text {uce }, i}\left(S_{i}^{*}\right)-\left[\Pi_{\mathcal{I}}^{*}\left(p_{\text {uce }}\right)-\Pi_{\mathcal{I} \backslash i}^{*}\left(p_{\text {uce }}\right)\right]
$$

where $\Pi_{L}^{*}(p)=\max _{S \in \Gamma} \sum_{i \in L} p_{i}\left(S_{i}\right)$ for bidders $L \subseteq \mathcal{I}$.

In the special case when prices are equal to valuations then this adjustment is equivalent to the standard definition of VCG payments.

\subsection{Informational Requirements}

Both CE and UCE prices have a central role in the preference elicitation problem. First, any auction that implements an efficient allocation must determine a set of CE prices. Second, any auction that implements the Vickrey outcome must determine a set of UCE prices. Segal (Chapter 11) provides an extended discussion.

Since the VCG auction is basically unique amongst the class of efficient auctions that take a zero payment from losing bidders (Ausubel and Milgrom, Chapter 1), these equivalences confirm the central role of prices in developing iterative CAs.

Theorem 4. (Parkes 2002, Nisan and Segal 2003) A combinatorial auction realizes the efficient allocation if and only if the auction also realizes a set of CE prices and an allocation supported in the price equilibrium. 
This result requires a technical condition of privacy-preservation, which precludes bidders from making their valuations contingent on the valuations of other bidders (e.g. "my value for $A$ is at least bidder 2's value for $A$ "). ${ }^{7}$

Theorem 5. (Parkes and Ungar 2002, Lahaie and Parkes 2004b) A combinatorial auction realizes the VCG outcome if and only if the auction also realizes a set of UCE prices and an allocation supported in the price equilibrium of the main economy.

That UCE prices provide sufficient information was first proved in Parkes and Ungar (2002). The necessary direction is due to Lahaie and Parkes (2004b). It is important to realize that the CE and UCE prices referenced in these results may only be realized implicitly and are not necessarily explicitly constructed in the auctions.

Considering minimal CE prices in particular, Mishra and Parkes (2004) note that minimal CE prices are universal iff BAS holds. In general, UCE prices are greater than the minimal $\mathrm{CE}$ prices because they must consider competition in the marginal economies in addition to the main economy.

The informational equivalence between the efficient outcome and the problem of discovering $\mathrm{CE}$ prices leads to a (largely negative) characterization of the worst-case communication complexity and preference-elicitation requirements of any efficient CA, iterative or otherwise (Segal, Chapter 11). On the other hand, iterative CAs are designed to have good elicitation properties on typical instances, while sealed-bid auctions must suffer the worst case every time. Moreover, this price equivalence suggests the central role of prices in the design of iterative CAs. Any protocol to determine the VCG outcome must (implicitly) determine UCE prices, so why not construct protocols to converge directly to UCE prices? We return to this theme in Section 4. 


\subsection{Examples}

The following examples illustrate the concept of CE and UCE prices and also serve to illustrate the principle that it is often unnecessary to receive complete information about bidder valuations to determine the Vickrey outcome. For each example, we define a space of valuations (that contain the true valuations) that provides sufficient information to determine the Vickrey outcome. The information is minimal — we call this a minimal information set - in the sense that no relaxed constraints on valuations are sufficient to pin down the Vickrey outcome.

\section{Example 2.1}

Consider a single-item auction with three bidders and values $(10,8,6)$. The efficient allocation assigns the item to bidder 1, and the Vickrey payment is $\$ 8$. Prices $10 \geq p \geq 8$ are all in CE, and $p=\$ 8$ is the unique anonymous UCE price. Notice that the UCE price must be at least $\$ 8$ to satisfy CE condition (1) for bidder 2 in $\operatorname{CAP}(\{1,2,3\})$ but no greater than $\$ 8$ to satisfy the same condition for bidder 2 in $\operatorname{CAP}(\{2,3\})$. The CE prices define a minimal information set, $\hat{\mathcal{V}}_{1}$, defined as the subset of valuations that satisfy constraints

$\left\{v_{1} \geq p, v_{2} \leq p, v_{3} \leq p, 10 \geq p \geq 8\right\}$. UCE prices imply additional information $\left\{v_{2}=8, v_{3} \leq 8\right\}$, which together with $\hat{\mathcal{V}}_{1}$ is a minimal information set for the VCG outcome. Notice that an ascending price (i.e. English) auction can elicit this information if bidders 1 and 2 bid up the price to just above 8 , at which point the auction terminates. Bidder 3 can remain silent.

\section{Example 2.2}

Consider a combinatorial allocation problem with items $\{A, B\}$ and 5 bidders (see Figure 2.1). The efficient allocation allocates $A$ to bidder 1 and $B$ to bidder 2 for a total value of 70 . The VCG payments are $p_{\mathrm{vcg}, 1}=30-(70-65)=25$ and $p_{\mathrm{vcg}, 2}=40-(70-55)=25$. Figure 2.1 (b) illustrates an information set on 
bidder valuations, that is sufficient to compute the VCG outcome and minimal in the sense that no constraints can be relaxed. The following prices are UCE for any valuation in this set: $p(A)=25, p(B)=25, p(A B)=25$ to bidders $\{1,2,4,5\}$ and prices $p_{3}(A)=20, p_{3}(B)=20, p_{3}(A B)=40$ to bidder 3 . In fact, these prices are also minimal CE prices and the discount computed in Eq. 4 is zero for bidders 1 and 2, and BAS is satisfied (because of the presence of bidders 4 and 5). Without these bidders, the BAS condition fails and the VCG payments become $p_{\mathrm{vcg}, 1}=0$ and $p_{\mathrm{vcg}, 2}=20$, which can be computed from UCE prices $p_{1}=(20,0,20), p_{2}=(0,40,40)$ and $p_{3}=(0,20,40)$. Additional information is needed from bidder 2 in this variation.

\section{The Design Space for Iterative Combinatorial Auctions}

The design space for iterative CAs is larger than for one-shot auctions. Important considerations include the design of information feedback to bidders and rules to guide the submission of bids. Cramton (Chapter 4) provides an in-depth discussion of many of these issues in the design of simultaneous ascending price auctions.

Let the state of an auction include all the information that is sufficient to define the future dynamics of the auction. For example, the state of an auction can define the ask prices, the provisional allocation, and also the bid improvement rules as they apply to particular bidders. Briefly, we can consider the role of the following design features:

Timing issues. Iterative auctions may be continuous, allowing bids to be submitted at any time with continual updates to the current provisional allocation and prices. Alternatively, iterative auctions may be discrete, or round-based, with the state updated periodically and with bidders provided with an opportunity to revise bids between rounds.

Continuous auctions can promote faster propagation of feedback information to bidders and help to quickly focus elicitation. However, 
continuous combinatorial auctions can be infeasible because the winner-determination problem must be resolved whenever a new bid is submitted. Continuous auctions also lead to high monitoring and participation costs for bidders. In comparison, discrete auctions allow an auctioneer to publish a schedule for rounds in the auction and bidders can plan when to allocate time to refine their values and bids.

Information feedback. Information feedback about the state of an auction can include information about the bids submitted and also aggregate information, such as price feedback and the current provisional allocation, to guide bidding. Information hiding is also possible, for example with rounding to limit the potential for signaling between bidders and with limited and discriminatory reporting of bid information.

Information feedback policies make a tradeoff between serving the goal of providing effective bid guidance and minimizing the opportunity for collusion and other forms of manipulation through signaling and coordination.

Bidding Rules. Ask prices are a common form of bid improvement rule, placing a lower-bound on the allowable bid price on a bundle. Bid improvement rules can also require a minimal percentage improvement over the current highest bid on a bundle, or over the total revenue in the next round given current bids. Activity rules (Milgrom 2000) introduce further restrictions, such as requiring that a bidder bids for a decreasing market share as prices increase during an auction. Ausubel, Cramton and Milgrom (Chapter 5) provide an extended discussion of bid-improvement and activity rules. Activity rules were introduced in the early FCC wireless spectrum auctions and proved important. ${ }^{8}$ Decisions about appropriate rules are often guided by a tradeoff between providing expressiveness so that bidders can follow straightforward bidding strategies, while promoting early information 
exchange between bidders and limiting the opportunity for bidders to wait and snipe at the end of an auction. Computational considerations also matter, for example linear prices can simplify the problem facing bidders in an auction (Kwasnica, Ledyard, Porter, and DeMartini 2004) but can be expensive to compute (Hoffman 2001).

Termination Conditions. Auctions may close at a fixed deadline, perhaps with an opportunity for a final sealed-bid round of bidding (sometimes called a proxy round). Alternatively, auctions can have a rolling closure with the auction kept open while one or more losing bidders continue to submit competitive bids.

Fixed deadlines are useful in settings in which bidders are impatient and unwilling to wait a long time for an auction to terminate. However, fixed deadlines tend to require stronger activity rules to prevent the auction reducing to a sealed-bid auction with bids delayed until the final round. In comparison, rolling closure rules have been shown to promote early and sincere bidding. ${ }^{9}$

Bidding Languages. A bid can be a complex object and expressed in terms of logical connectives (Nisan, Chapter 9). One popular bidding language is exclusive-or (XOR), in which bid $\left(p_{1}, S_{1}\right)$ xor $\left(p_{2}, S_{2}\right)$ xor $\ldots$ xor $\left(p_{l}, S_{l}\right)$ has semantics "I will buy at most one of these bundles" at the stated bid price. Another popular language is additive-or (OR) bidding languages, in which bid $\left(p_{1}, S_{1}\right)$ or $\left(p_{2}, S_{2}\right)$ or $\ldots$ or $\left(p_{l}, S_{l}\right)$ has semantics "I will buy one or more of these bundles" at the stated bid price. Bidding languages can also place constraints on the bid prices, for example by requiring click-box bidding in which bidders must submit bids from a menu. ${ }^{10}$ The expressiveness of a bidding language in an iterative CA must be considered together with the opportunity to refine bids during an auction. For instance, a language that is additive-or on items is not expressive in a 
one-shot CA but becomes expressive in an ascending auction when bidders can decommit from bids. ${ }^{11}$ Bidding languages are often designed to support straightforward bidding with bidders able to state the bundle that maximizes their surplus in response to prices in each round.

Proxy agents. Proxy agents provide a still richer interface for iterative CAs (Parkes and Ungar 2000b, Ausubel and Milgrom 2002). Bidders can provide direct value information to an automated bidding agent that bids on their behalf within an auction. The bidder-to-proxy language should allow a bidder to express partial and incomplete information, to be refined during the auction, in order to realize the elicitation and price discovery benefits of an iterative auction.

Proxy agents can query a bidder actively when they have insufficient information to submit bids. Proxy agents can also facilitate faster convergence with rapid automated proxy rounds interleaved with bidder rounds. Mandatory proxy agents can be useful in restricting the strategy space available to bidders.

One concern in the design of proxy auctions is to determine when to allow proxy information to be revised and to determine the degree of consistency to enforce across revisions. An additional concern is that of trust and transparency since the bidding activity is transferred to automated agents.

\section{Price-Based Iterative Combinatorial Auctions}

Many iterative CAs are price based and provide ask prices to guide bidding. In this section we survey some of these auction designs. We limit our attention to auctions designed for valuations that are rich enough to include the substitutes valuations. As such, we exclude the assignment model in which bidders have unit-demand for items. See Bikhchandani and Ostroy (Chapter 8) for a taxonomy that includes this case. 
All the auctions that we discuss share the same high level structure:

In each round the auctioneer announces ask prices and a provisional allocation and requests new bids from bidders. The bids are used to formulate a new winner-determination problem and update the provisional allocation, and also to adjust ask prices and test for termination.

Table 2.1 provides a summary of the characteristics of some well-known auctions, stating properties for straightforward (non-strategic) bidding. For the cases in which an auction terminates with the VCG outcome this assumption is justified in an ex post equilibrium but otherwise one should expect incentives for demand reduction. The auctions are described in terms of the structure of the price space, the bidding language, and the method used to update prices. 


\begin{tabular}{cccccc}
\hline \hline Name & Valuations & Price structure & $\begin{array}{c}\text { Bidding } \\
\text { language }\end{array}$ & $\begin{array}{c}\text { Price Update } \\
\text { method }\end{array}$ & Outcome \\
\hline KC & substitutes & non-anon items & OR-items & greedy & CE \\
SAA & substitutes & items & OR-items & greedy & CE \\
GS & substitutes & items & XOR & minimal & min CE $^{12}$ \\
Aus & substitutes & items & single & greedy $^{a}$ & VCG \\
\hline$i$ Bundle; Ascending-proxy & BSM & non-anon bundles & XOR & greedy & VCG \\
$\ldots$ & general & $\ldots$ & $\ldots$ & $\ldots$ & min CE \\
dVSV & BSM & non-anon bundles & XOR & minimal & VCG \\
Clock-proxy & BSM & items $(+ \text { proxy })^{c}$ & XOR & greedy & VCG \\
$\ldots$ & general & $\ldots$ & $\ldots$ & $\ldots$ & min CE \\
\hline RAD & general & items & OR & LP-based & - \\
A $k$ BA & general & anon bundles & XOR & LP-based & - \\
BEA & general & non-anon bundles & XOR & greedy & VCG \\
MP & general & non-anon bundles & XOR & minimal & VCG \\
\hline \hline
\end{tabular}

Table 2.1: Price-Based Combinatorial Auctions. Formal properties are stated for straightforward bidding, and with the most general class of valuations for which the property holds. Notation '- ' in the Outcome column indicates that no formal properties have been established.

Notes:

a Aus traces $n+1$ trajectories.

$b \quad$ Ascending-proxy dynamics are identical to $i$ Bundle(3), although ascending-proxy emphasizes a sealed-bid proxy auction form.

$c$ Clock-proxy is a hybrid design, with a linear-price clock auction followed by a sealed-bid ascending-proxy auction.

$d$ Ascending price while the auction is open, followed by a downwards adjustment after termination.

Abbreviations:

KC (Kelso and Crawford 1982) SAA (Milgrom 2000)

GS (Gul and Stacchetti 2000)

$i$ Bundle (Parkes and Ungar 2000a)

Aus (Ausubel 2002)

dVSV (de Vries, Schummer, and Vohra 2003)

RAD (Kwasnica et al. 2003)

$i$ BEA (Parkes and Ungar 2002)

Ascending-proxy (Ausubel and Milgrom 2002)

Clock-proxy (Ausubel and Milgrom, Chapter 5)

A $k$ BA (Wurman and Wellman 2000)

MP (Mishra and Parkes 2004)

We see a wide variety of prices, from simple prices on items (linear prices) to non-anonymous prices on bundles (non-anonymous and non-linear). In addition, the auctions vary in the bids that a bidder can submit in each round: OR-items, an additive-or bid for multiple items; XOR, an exclusive-or bid for multiple bundles; single, a bid on a single bundle in each round; $O R$, an additive-or bid for multiple 
bundles. The XOR language has emerged as the definitive choice in recent designs.

The price-update methods, which characterize the rules by which prices are computed in each round, are broken down as follows:

Greedy update: The price is increased on some arbitrary set (perhaps all) of the over-demanded items or bundles.

Minimal update: The price is increased on a minimal set of overdemanded items, or based on the bids from a set of minimally undersupplied bidders.

LP-based: A linear program, formulated to find prices that are good approximations to $\mathrm{CE}$ prices given current bids, is used to adjust prices.

For linear prices, Demange, Gale and Sotomayor (1986) in the assignment model and later Gul and Stacchetti (2000) for substitutes define a minimal update in terms of increasing the prices on a minimal overdemanded set of items. ${ }^{13}$ Minimal price updates are adopted to drive prices towards minimal CE prices. de Vries, Schummer and Vohra (2003) generalize this to define updates in terms of minimally undersupplied bidders ${ }^{14}$ and define a minimal update for general CAs. All bidders in a minimally undersupplied set face higher prices on the bundles for which they submitted a bid.

$\mathrm{RAD}$ and $\mathrm{A} k \mathrm{BA}$ adopt LP-based price updates and adjust prices to find good approximations to $\mathrm{CE}$ prices given current bids and the current provisional allocation. RAD seeks linear and anonymous prices while $\mathrm{A} k \mathrm{BA}$ seeks non-linear but anonymous price approximations. Formal convergence properties have not been proved for $\mathrm{RAD}$ or $\mathrm{A} k \mathrm{BA}$, although $\mathrm{RAD}$ reduces to a simultaneous ascending price auction for substitutes valuations.

The auctions that are able to implement the VCG outcome (for instance, Aus for substitutes and dVSV for BSM coalitional values) are interesting because they bring straightforward bidding into an equilibrium. Straightforward bidding is a 
best response, whatever the valuations of other bidders, as long as the other bidders also follow a straightforward (perhaps untruthful) bidding strategy. This ex post equilibrium concept is useful because it places no requirements on the knowledge that bidders have of the valuations of other bidders.

Winning bidders pay their final bid price in all auctions except Aus, $i$ BEA and MP. Aus allows for $(n+1)$ restarts and uses information elicited along each trajectory to determine the final payments. $i$ BEA and MP terminate with UCE prices, at which point final payments are determined through downwards adjustments. Auction clock-proxy (Ausubel, Cramton and Milgrom Chapter 5) is a hybrid auction. The first stage maintains item prices and runs an ascending-clock CA (see also Porter, Rassenti, and Smith (2003)). This stage is used for price discovery and can be considered to construct approximate linear CE prices. The second stage is sealed-bid, with bids from the first stage combined with additional bids that must be consistent with bids from the clock phase.

\subsection{Insufficiency of Simple Prices}

It is interesting to consider what form of prices are necessary to implement efficient ascending CAs. Gul and Stacchetti (2000) first addressed this question, in the setting of substitutes valuations. The authors provide a formal definition of an ascending CA, but limit attention to linear and anonymous prices. They show that there exists no ascending VCG auction with linear and anonymous prices for substitutes valuations. The auction due to Ausubel (2002) lies outside of this negative characterization because it uses $n+1$ price trajectories.

Recently, Mishra and Parkes (2004) used the UCE-based price characterization to demonstrate that efficient ascending CAs require both non-anonymous and non-linear prices, even for this case of substitutes valuations. The authors exhibit instances for which only non-anonymous and non-linear UCE prices exist. As for sufficiency, auctions dVSV and $i$ Bundle are examples of ascending VCG auctions for substitutes valuations that maintain these rich prices. 
However, de Vries, Schummer, and Vohra (2003) extend the definition of ascending CAs in Gul and Stacchetti (2000) to allow for non-anonymous and non-linear prices and obtain a negative result. When at least one bidder has a non-substitutes valuation an ascending CA cannot implement the VCG outcome even when the other bidders are restricted to substitutes and even with non-anonymous and non-linear prices. Auctions $i$ BEA and MP lie outside of this negative characterization because they allow a final downwards adjustment to determine final prices.

Thus, with substitutes values but simple prices we must accept auctions with multiple trajectories or non-monotonic adjustments. Moreover, although rich prices extend the reach of ascending CAs to substitutes values we still need to accept multiple trajectories or non-monotonic adjustments to handle richer preferences than substitutes.

\subsection{Primal-Dual Auction Design}

Many traditional combinatorial optimization problems can be solved with primal-dual algorithms. A primal-dual approach uses linear-programming (LP) duality to formulate an optimization problem as a satisfaction problem. Strong LP duality states that a pair of feasible primal and dual solutions are optimal if and only if they satisfy complementary slackness (CS) conditions. We provide a brief review of LP theory at the end of this chapter, and refer the reader to Papadimitriou and Steiglitz (1998) for a textbook treatment.

In fact, primal-dual theory also provides a useful conceptual framework for the design of iterative price-based CAs. Prices are interpreted as a feasible dual solution and the provisional allocation is interpreted as a feasible primal solution. Bids provide sufficient information to formulate and solve restricted primal and dual problems, the winner-determination and price-update problems respectively (see Figure 2.2). For further discussion of this idea, see Parkes (2001), de Vries, Schummer and Vohra (2003) and Bikhchandani and Ostroy (Chapter 8). 
Straightforward bidding is first assumed, and later justified by termination with VCG payments. The winner-determination problem uses information implicit in bids to compute a feasible solution that minimizes the violation of the CS conditions, and price updates adjust the dual solution towards an optimal dual solution. ${ }^{15} \mathrm{CS}$ conditions have an exact equivalence with conditions (1) and (2) required for $\mathrm{CE}$ prices, and are satisfied on termination of an auction. Constructively, primal-dual auction design requires the following steps:

1. Formulate an LP for the CAP that is integral, such that its optimal solution is a feasible allocation. The dual problem should allow convergence to UCE prices, or to minimal CE prices that support VCG payments in the case of BAS valuations.

2. Provide bidders with a bidding language that is expressive for straightforward bidding, and formulate a winner-determination problem to compute a feasible primal solution that minimizes the violation of CS conditions as represented in bids.

3. Terminate when the provisional allocation and ask prices satisfy CS conditions (and thus represent a CE), and also satisfy any additional conditions that are necessary to compute the VCG payments at termination (e.g. UCE conditions or minimal CE prices). Otherwise, adjust prices to make progress towards an optimal dual solution that satisfies these conditions.

The characterization of VCG payments in terms of minimal CE and UCE prices suggests two methods to adjust towards the VCG outcome. The methods are illustrated in Figure 2.3, which considers the price on bundles, $S_{1}$ and $S_{2}$, allocated to bidders 1 and 2 in the efficient outcome.

In case (a), the coalitional value function satisfies BSM and the VCG payments are supported at the minimal CE prices. Ascending CAs (such as dVSV) can 
converge monotonically to these prices and the VCG outcome. In case (b), the coalitional value function satisfies neither BSM not BAS. Although each bidder's VCG payment is supported in some minimal CE there is no single CE that supports the VCG payment to both bidders simultaneously. As illustrated, ascending CAs such as $i \mathrm{BEA}$ and MP can still converge monotonically to UCE prices from which the VCG outcome can be determined in a final adjustment. The next section presents a case study of primal-dual methods to the design and analysis of the $i$ Bundle auction. ${ }^{16}$ In Section 4.4 we return to the auctions in Table 2.1, and discuss each in a little more detail.

\subsection{Case Study: $i$ Bundle}

We will focus on variation $i \operatorname{Bundle}(2)$, in which prices are non-linear but anonymous. This variation is efficient with straightforward bidding and an additional requirement that bidder strategies satisfy a "safety" property. Later, we also briefly describe $i$ Bundle(3), which employs non-linear and non-anonymous prices and is efficient without the safety condition.

The interested reader is referred to Parkes and Ungar (2000a) and Parkes (2001) for additional details, including a description of $i$ Bundle(d), which blends $i$ Bundle(2) and $i$ Bundle(3) and allows for dynamic price discrimination decisions to be made during the auction. In what follows, we will use $i$ Bundle to refer to variation $i$ Bundle(2) unless otherwise stated.

\section{$i$ Bundle(2): Anonymous Prices}

$i$ Bundle maintains ask prices on bundles and a provisional allocation and proceeds in rounds, indexed $t \geq 1$. In each round a bidder can submit XOR bids on bundles. In general the bid price on a bundle must be at least the ask price. Bidders must resubmit bids on any bundle that they are winning in the current provisional allocation but can bid at the same price on such a bundle even if the ask price has since increased. A bidder can also bid at $\epsilon$ less than the ask price 
when making a "last-and-final" bid, at which point she can no longer improve her price. Equivalently, one can simply retain all bids from previous rounds. A bid at, or above, the current ask price is said to be competitive, and a bidder is competitive if she submits at least one competitive bid.

The winner-determination problem in each round is to compute a provisional allocation to maximize the seller's revenue given bids, with at most one bundle selected from the XOR bid of each bidder. Let $\mathcal{B}_{i}$ denote the bids from bidder $i$, and $p_{\text {bid, } i}(S)$ denote the bid price on bundle $S \in \mathcal{B}_{i}$. Winner determination can be formulated as the following mathematical program:

$$
\begin{aligned}
\max _{x_{i}(S)} & \sum_{i \in \mathcal{I}} \sum_{S \in \mathcal{B}_{i}} x_{i}(S) p_{\mathrm{bid}, i}(S) \\
\text { s.t. } \quad \sum_{S \in \mathcal{B}_{i}} x_{i}(S) & \leq 1, \quad \forall i \\
\sum_{i \in \mathcal{I}} \sum_{S \in \mathcal{B}_{i}: j \in S} x_{i}(S) & \leq 1, \quad \forall j \\
x_{i}(S) & \in\{0,1\}, \quad \forall i, \forall S \in \mathcal{B}_{i}
\end{aligned}
$$

Constraint (5) restricts the seller to selecting at most one bid from each bidder. Constraint (6) ensures the allocation is feasible. Ties are broken first to favor the allocation from the previous round and then to maximize the number of winning bidders.

$i$ Bundle terminates when each competitive bidder receives a bundle in the provisional allocation. Otherwise, prices are increased, by $\epsilon$ above the bid price on all bundles that receive a bid from some losing bidder in the current round and the new allocation and prices are provided as feedback to bidders. Prices on other bundles are implicitly adjusted to satisfy free disposal, although only bundles that receive losing bids need to be explicitly quoted. On termination the provisional allocation becomes the final allocation, and bidders pay their final bid prices. $i$ Bundle maintains feasible primal and dual solutions to an extended LP formulation of CAP and terminates with a CE outcome that satisfies CS 
conditions. The proof technique is inspired by Bertsekas' (1987) analysis of the AUCTION algorithm for the special case of unit-demand valuations.

Given ask prices, $p_{i}(S)$, to bidder $i$ we define $\epsilon$ straightforward bidding in terms of an $\epsilon$-demand set, $\epsilon$-DS, which is:

$$
\epsilon D_{i}\left(p_{i}\right)=\left\{S: v_{i}(S)-p_{i}(S)+\epsilon \geq \max _{S^{\prime}}\left(v_{i}\left(S^{\prime}\right)-p_{i}\left(S^{\prime}\right), 0\right), \forall S \subseteq \mathcal{G}\right\}
$$

In words, bidders state in their bid all bundles that come within $\epsilon$ of maximizing their surplus given prices in each round. This reduces to straightforward bidding for a small enough $\epsilon$.

Definition 5 (Safety). The competitive bundles in the $\epsilon$-demand set of each losing bidder in each round are non-disjoint, i.e. each pair of bundles shares at least one item.

For example, losing bids $\{(A B C, \$ 100),(C D E, \$ 50)\}$ from a single bidder satisfy safety, while losing bids $\{(A B C, \$ 100),(D E, \$ 50)\}$ from a single bidder fail the safety condition.

Theorem 6. (Parkes and Ungar 2000a) iBundle(2) terminates with an allocation that is within $3 \min (n, m) \epsilon$ of the efficient solution for $\epsilon$-straightforward bidding strategies and with bid safety.

The first step of the proof is to introduce an extended LP formulation $\left(\mathrm{LP}_{2}\right)$ for CAP due to Bikhchandani and Ostroy (2002, see also Chapter 8). $\mathrm{LP}_{2}$ is integral when the safety condition holds for straightforward bidding. The dual formulation $\left(\mathrm{DLP}_{2}\right)$ has variables that correspond to anonymous and non-linear prices. Let $K$ denote the set of feasible partitions. For example, $(A, B, C)$ and $(A B, C)$ are feasible partitions for items $A B C$. Variable $y(k)=1$ will indicate that the allocation must be restricted to bundles in partition $k \in K$. For example, if partition $(A B, C)$ is selected then the only valid allocations are those in which 
$A B$ goes to some bidder and $C$ to another bidder. We have:

$$
\begin{gathered}
\max _{x_{i}(S), y(k)} \sum_{S \subseteq \mathcal{G}} \sum_{i \in \mathcal{I}} x_{i}(S) v_{i}(S) \\
\text { s.t. } \quad \sum_{S \subseteq \mathcal{G}} x_{i}(S) \leq 1, \quad \forall i \\
\sum_{i \in \mathcal{I}} x_{i}(S) \leq \sum_{k \in K: S \in k} y(k), \quad \forall S \\
\sum_{k \in K} y(k) \leq 1 \\
x_{i}(S), y(k) \geq 0, \quad \forall i, S, k \\
\min _{\pi_{i}, p(S), \Pi_{s}} \sum_{i \in \mathcal{I}} \pi_{i}+\Pi_{s} \\
\pi_{i}+p(S) \geq v_{i}(S), \quad \forall i, S \\
\Pi_{s}-\sum_{S \in k} p(S) \geq 0, \quad \forall k \\
\pi_{i}, p(S), \Pi_{s} \geq 0, \quad \forall i, S
\end{gathered}
$$

Dual variable $p(S)$ can be interpreted as the ask price on bundle $S$. Then, optimal $\pi_{i}^{*}=\max _{S}\left\{v_{i}(S)-p(S), 0\right\}$ defines the maximal payoff to bidder $i$ across all bundles given prices, and optimal $\Pi_{s}^{*}=\max _{k \in K} \sum_{S \in k} p(S)$ defines the maximal revenue to the seller across all partitions given prices. This is also the maximal revenue across all allocations because prices are anonymous.

The dual problem sets prices to minimize the sum of the maximal payoff to each bidder and the maximal revenue to the seller. Optimal dual prices will correspond to $\mathrm{CE}$ prices whenever the primal LP is integral.

Interpret the provisional allocation and ask prices in a round of $i \operatorname{Bundle}(2)$ as defining a feasible primal and a feasible dual solution (denoted $\hat{x}, \hat{y}, \hat{\pi}_{i}, \hat{p}$, and $\hat{\Pi}_{s}$ ). We can now establish termination with CS conditions for straightforward bidding strategies.

The first primal CS condition is:

$$
\hat{x}_{i}(S)>0 \Rightarrow \hat{\pi}_{i}+\hat{p}(S)=v_{i}(S), \quad \forall i, S
$$


This states that any bundle allocated to bidder $i$ must maximize her payoff across all bundles at the prices. Condition (CS-1) is approximately satisfied in every round because the provisional allocation is selected with respect to bids, which are in turn drawn from $\epsilon$ demand sets. Formally, a relaxed form of condition (CS-1) holds, with $\hat{x}_{i}(S)>0 \Rightarrow \hat{\pi}_{i}+\hat{p}(S) \leq v_{i}(S)+2 \epsilon$, for all $i$ and $S$.

The second primal CS condition is:

$$
\hat{y}(k)>0 \Rightarrow \hat{\Pi}_{s}-\sum_{S \in k} \hat{p}(S)=0, \quad \forall k
$$

This states that the provisional allocation must maximize the seller's payoff (i.e. revenue) given the prices, across all feasible allocations and irrespective of bids received from bidders.

Bundle $S$ has a strictly positive price if it is greater than the price on every bundle contained in $S$. Then, (CS-2) follows from properties (P1) and (P2), which are maintained in each round of the auction:

(P1) All bundles with strict positive prices receive a bid from some bidder in every round.

(P2) One or more of the revenue-maximizing allocations in every round can be constructed from bids from different bidders.

Formally, (P1) follows because one can show that a losing bidder will continue to bid for $S$ in the next round, even at the higher price. Property (P2) follows from the safety property, which prevents a single bidder from causing the price to increase on a pair of disjoint bundles. This is why we need the safety condition. Combining (P1) and (P2), and together with $\epsilon$-DS, we get a relaxed formulation of (CS-2), with $\hat{y}(k)>0 \Rightarrow \hat{\Pi}_{s}-\sum_{S \in k} \hat{p}(S) \leq \min (m, n) \epsilon$, for all partitions $k \in K$.

Dual CS condition (CS-3), states:

$$
\hat{\pi}_{i}>0 \Rightarrow \sum_{S \subseteq G} \hat{x}_{i}(S)=1, \quad \forall i
$$


In words, every bidder with positive payoff for some bundle at the current prices must receive a bundle in the provisional allocation. (CS-3) is satisfied for all bidders that receive bundles in a particular round, but not for the losing bidders that are still competitive. However, (CS-3) holds for every bidder on termination because at this point $\epsilon-\mathrm{DS}=\emptyset$ for all losing bidders.

(CS-3) and (CS-1) are equivalent to CE condition (1) and (CS-2) together with an additional requirement that a provisional allocation is always selected is equivalent to $\mathrm{CE}$ condition (2).

Finally, we obtain an upper-bound on the worst-case efficiency error of $i$ Bundle, in terms of the minimal bid increment $\epsilon$. First, sum the approximate (CS-1) condition over all bidders in the final allocation, and substitute $\hat{\pi}_{i}=0$ for bidders not in the allocation by (CS-3). This gives:

$$
\begin{array}{r}
\sum_{i \in \mathcal{I}} \hat{\pi}_{i} \leq \sum_{i \in \mathcal{I}} v_{i}\left(\hat{S}_{i}\right)-\sum_{i \in \mathcal{I}} \hat{p}\left(\hat{S}_{i}\right)+2 \min (m, n) \epsilon \\
\Rightarrow \hat{\Pi}_{s}+\sum_{i \in \mathcal{I}} \hat{\pi}_{i} \leq \sum_{i \in \mathcal{I}} v_{i}\left(\hat{S}_{i}\right)+3 \min (m, n) \epsilon
\end{array}
$$

where Eq. (7) follows because an allocation can include no more bundles than there are items or bidders, and Eq. (8) is by substitution of the $\epsilon$-approximate (CS-2) condition.

The LHS of Eq. (8) is the value of the final dual solution, and the first-term on the RHS is the value of the final primal solution. Now, $\hat{\Pi}_{s}+\sum_{i} \hat{\pi}_{i} \geq w(\mathcal{I})$, (the value of the optimal primal) by LP weak duality, and therefore $w(\mathcal{I}) \leq \hat{\Pi}_{s}+\sum_{i} \hat{\pi}_{i} \leq \sum_{i} v_{i}\left(\hat{S}_{i}\right)+3 \min (m, n) \epsilon$.

A complete proof must also show termination. The basic idea is to assume the auction never terminates and prove that a bidder must eventually submit a bid at a price above her valuation, assuming finite values and a finite number of items, from which we get a contradiction with straightforward bidding. 


\section{iBundle(3): Non-anonymous Prices}

$i$ Bundle(3) is the variation of $i$ Bundle in which each bidder faces non-anonymous prices in every round. The dynamics of $i$ Bundle(3) with straightforward bidding are identical to that of Ausubel and Milgrom's (2002) ascending-proxy auction, although ascending-proxy is not described in price terms. $i$ Bundle(3) is efficient for straightforward bidding with general values. Moreover, the auction will terminate with VCG outcomes for BSM coalitional value functions.

Let $p_{\text {ask }, i}^{t}(S)$ denote the ask prices to bidder $i$ in round $t$. Initially, $p_{\text {ask }, i}^{1}(S)=0$ for all bundles $S$ and all bidders. Bids are received, and the winner determination problem solved, as in $i$ Bundle(2). Then, for each bidder not in the provisional allocation, the price to that bidder is increased by the minimal bid increment, $\epsilon>0$, above her bid price on all bundles submitted in that round, and adjusted for free-disposal.

It is now quite immediate to establish that $i \mathrm{Bundle}(3)$ terminates in $\mathrm{CE}$ with straightforward bidding. The prices faced by a bidder in round $t$ are parameterized by $\pi_{i}^{t} \geq 0$, which can be interpreted as the maximal payoff to the bidder in that round. The ask price on bundle $S$ in round $t$ is defined as:

$$
p_{\text {ask }, i}^{t}(S)=\max \left(0, v_{i}(S)-\pi_{i}^{t}\right)
$$

Initially, $\pi_{i}^{1}=\max _{S}\left\{v_{i}(S)\right\}$, for all $i$, and the price is zero on all bundles. The payoff $\pi_{i}^{t}$ decreases monotonically during the auction and prices monotonically increase. The $\epsilon$-DS for bidder $i$ in round $t$ includes every bundle for which $v_{i}(S) \geq \pi_{i}^{t}$, and increases monotonically across rounds. Eventually, when $\pi_{i}^{t}$ is less than $\epsilon$ the prices on each bundle are within $\epsilon$ of her value and she will bid for every bundle with positive value in her $\epsilon$-DS. ${ }^{17}$

Condition (CS-1) holds trivially in each round and (CS-3) holds at termination, just as in $i$ Bundle(2). In addition, (CS-2) holds in each round because of the special structure of prices: every bundle with a strict positive price receives a bid in a bidder's $\epsilon$-DS. This does not require the safety condition. 
Theorem 7. (Parkes and Ungar 2000a) iBundle(3) terminates with an allocation that is within $3 \min (n, m) \epsilon$ of the efficient allocation for $\epsilon$-straightforward bidding strategies and with bid safety.

Theorem 8. (Ausubel and Milgrom 2002) iBundle(3) terminates with minimal CE prices and the VCG outcome for BSM valuations and straightforward bidding.

Proof. Consider an arbitrary bidder $j$, and let $\bar{\pi}_{j}$ denote her payoff in the minimal CE prices. Refer to the bidders in the provisional allocation in round $t$ as the winning coalition. We prove that the payoff, $\pi_{j}^{t}$ to bidder $j$ in any round $t$ satisfies $\pi_{j}^{t} \geq \bar{\pi}_{j}$. First, bidder $j$ must be in the winning coalition in any round in which $\pi_{j}^{t}<\bar{\pi}_{j}$. To see this, consider a coalition $L \subset \mathcal{I}$, with $j \notin L$, and observe that the revenue to the seller from coalition $L$ in round $t$ is exactly $w(L)-\sum_{i \in L} \pi_{i}^{t}$ from Eq. (9). Then,

$$
\begin{aligned}
w(L)-\sum_{i \in L} \pi_{i}^{t} & <w(L)-\sum_{i \in L} \pi_{i}^{t}+\left(\bar{\pi}_{j}-\pi_{j}^{t}\right) \\
& =w(L)-\sum_{i \in L \cup\{j\}} \pi_{i}^{t}+w(\mathcal{I})-w(\mathcal{I} \backslash j) \\
& \leq w(L)-\sum_{i \in L \cup\{j\}} \pi_{i}^{t}+w(L \cup\{j\})-w(L) \\
& =w(L \cup\{j\})-\sum_{i \in L \cup\{j\}} \pi_{i}^{t}
\end{aligned}
$$

where Eq. (10) follows from the equivalence between maximal payoff and VCG payoff for BSM valuations and Eq. (11) follows from the BSM condition. Thus, the payoff to bidder $j$ cannot fall more than $\epsilon$ below $\bar{\pi}_{j}$ (since the bidder always wins, and its prices are unchanged), and prices converge to the minimal CE prices as $\epsilon \rightarrow 0$.

An ex post equilibrium is invariant to the values of bidders, i.e. straightforward bidding is an equilibrium even ex post once every bidder knows the values of other bidders. 
Theorem 9. Straightforward bidding is an ex post equilibrium of iBundle(3), and the auction is efficient, for BSM valuations.

This result requires that the revealed preferences by a bidder are consistent with some valuation during the auction. ${ }^{18}$ Given this, we can fix the reports $v_{-i}$ of other bidders. If bidder $i$ follows a straightforward strategy the auction implements the VCG outcome because valuations satisfy BSM. Moreover, if bidder $i$ reports some other valuation $\hat{v}_{i} \neq v_{i}$ the auction implements the efficient allocation for $\left(\hat{v}_{i}, v_{-i}\right)$ and CE prices that are at least the bidder's Vickrey payment in that outcome. Thus, bidder $i$ 's best-response is straightforward bidding because her payoff in the truthful Vickrey outcome dominates her payoff in any other Vickrey outcome, and therefore also in this alternate CE outcome.

\subsection{Ascending Price Combinatorial Auctions}

Perhaps the defining feature of the $i$ Bundle family of auctions is that they allow non-linear, and sometimes non-anonymous ask prices. Only the dVSV, $i$ BEA and MP auctions have a similarly rich class of prices. The other auctions in Table 2.1 maintain simpler prices, typically anonymous and often linear. In describing the auctions we group together auctions $\mathrm{KC}, \mathrm{SAA}, \mathrm{GS}$ and Aus because they are all designed to handle the special case of substitutes valuations. Then we briefly discuss dVSV, which is designed for a BSM coalitional value function, and is presented in detail in Bikhchandani and Ostroy (Chapter 8). The ascending-proxy auction is a sealed-bid implementation of $i$ Bundle(3) with interesting theoretical properties, and will be discussed along with other proxied auctions in Section 5.2 and presented in more detail in Ausubel and Milgrom (Chapter 3). Finally, we describe the clock-proxy, iBEA and MP auctions, which are designed for general valuations. 


\section{Special-Case: Goods are Substitutes}

Recall that linear CE prices exist for substitutes valuations, but that non-linear and non-anonymous prices are still required to support VCG payments, even for substitutes.

Auction $\mathrm{KC}$ was first described in the setting of a matching problem, with multiple firms and multiple workers. The matching problem can be reinterpreted as an allocation problem with each firm corresponding to a bidder and each worker to an item. Bidders can submit bids for multiple items in each round. Winner determination allocates all items that receive bids and prices are increased on over-demanded items. The auction converges to a competitive equilibrium outcome and an efficient allocation for straightforward bidding. Kelso and Crawford (1982) do not investigate strategic behavior or the relationship between the outcome and the VCG payoffs.

Auction SAA is closely related to $\mathrm{KC}$ in that bidders can submit bids for multiple items and the bid on an item must be repeated if it is winning. However, SAA maintains anonymous prices and is distinguished in its careful use of activity and bid-improvement rules. The auction design forms the basis of the series of FCC wireless spectrum auctions.

Auction GS adopts the same basic methodology as KC, except that prices are anonymous and increased on a set of minimal overdemanded items. This provides termination with minimal CE prices when bidders are straightforward. Just as in $\mathrm{KC}$ and SAA, these prices do not support the VCG outcome for substitutes valuations and straightforward bidding is not an equilibrium.

Auction Aus is unique amongst the auctions for substitutes valuations in its ability to terminate with the Vickrey outcome. Ausubel (2002) achieves this despite using only anonymous item prices by running $n+1$ separate auctions, each with its own price trajectory. Information across each auction is used to adjust final payments to VCG payments. Let $\left(\mathcal{A}_{-1}, \ldots, \mathcal{A}_{-n}, \mathcal{A}\right)$, denote the sequence of auctions in Aus, with bidder $i$ excluded from participation in auction $\mathcal{A}_{-i}$. All 
bidders are invited to participate in the final auction. The allocation is determined in auction $\mathcal{A}$, but the payment by bidder $i$ is determined from the price and bidding dynamics in auctions $\mathcal{A}_{-i}$ and $\mathcal{A}$. The dynamics in $\mathcal{A}_{-i}$ are used to adjust downwards the final payment for bidder $i$.

\section{Bidder Submodular}

Auction dVSV is similar to $i$ Bundle, with bids for XOR sets of bundles and prices that are non-linear and non-anonymous and increased based on bids from losing bidders. However, the price update rule is different. dVSV increases prices on the set of minimally-undersupplied bidders. This set can include bidders that are in the current provisional allocation, as well as losing bidders, and is different from the set of losing bidders on which prices are adjusted in $i$ Bundle. Although there has been no computational study, de Vries, Schummer, and Vohra (2003) argue by analogy to algorithms in the optimization literature that dVSV will converge more quickly than $i$ Bundle. ${ }^{19}$ In $i$ Bundle's favor is that the price-update step is simple to explain to bidders and easy to compute.

\section{General-Purpose CAs}

$\mathrm{RAD}$ and $\mathrm{A} k \mathrm{BA}$ are general-purpose ascending $\mathrm{CAs}$, designed without restrictions on agent valuations. Although an equilibrium analysis is not available for either auction their performance has been evaluated experimentally, through human-based laboratory studies and through computational simulation. Both auctions formulate an LP to adjust prices. AkBA provides non-linear prices and supports an XOR bidding language while RAD provides linear prices and supports an OR bidding language.

A competitive equilibrium perspective provides a unifying view of the auctions. Recall that CE prices in CAP must be both non-linear and non-anonymous in general. One can interpret $\mathrm{A} k \mathrm{BA}$ as an iterative procedure to determine anonymous and non-linear prices that approximate CE prices, and RAD as an 
iterative procedure to determine anonymous and linear prices that approximate CE prices.

The bidding rules and winner-determination step in $\mathrm{A} k \mathrm{BA}$ are much as in $i$ Bundle. Each bidder submits an XOR bid, from which the winner-determination problem is formulated. A $k \mathrm{BA}$ differs from $i$ Bundle in the price-update step, which is parameterized with $0 \leq k \leq 1$.

Let $S^{t}=\left(S_{1}^{t}, \ldots, S_{n}^{t}\right)$ denote the provisional allocation in round $t, p_{\text {ask }}^{t}(S)$ denote the ask price on $S, \Delta^{t}\left(S^{\prime \prime}, S^{\prime}\right)=p^{t}\left(S^{\prime \prime}\right)-p^{t}\left(S^{\prime}\right)$ denote the price difference between bundle $S^{\prime \prime}$ and bundle $S^{\prime}, W^{t}$ denote the current winners, and $D S_{i}\left(p_{\text {ask }}^{t}\right)$ denote the bids submitted by bidder $i$ in response to ask prices. AkBA computes prices for period $t+1$ that will maintain CS condition (CS-1) for all bidders, given the demand-set information in their most recent bid. In particular, prices $p_{\text {ask }}^{t+1}(S)$ are computed to satisfy: a) $p_{\text {ask }}^{t+1}(S) \geq p^{t}(S)$, for all bundles $S \in S^{t}$ that receive bids from some losing bidder, $i \notin W^{t}$.

b) $\Delta^{t+1}\left(S^{\prime \prime}, S^{\prime}\right) \geq \Delta^{t}\left(S^{\prime \prime}, S^{\prime}\right)$ for any pair of bundles $S^{\prime \prime}, S^{\prime}$, such that $S^{\prime}$ is allocated to a winning bidder $i \in W^{t}$, and that bidder also bids on $S^{\prime \prime}$.

These prices are not unique in general, and $\mathrm{A} k \mathrm{BA}$ breaks the tie by selecting a convex combination of prices, with $p_{\text {ask }}^{t+1}(S)=(1-k) \underline{p}^{t+1}(S)+k \bar{p}^{t+1}(S)$, where $\underline{p}^{t+1}(S)$ and $\bar{p}^{t+1}(S)$ are the minimal and maximal prices that satisfy conditions a) and b), for some parameter $0 \leq k \leq 1$.

Finally, new bids must improve the price by a minimal bid increment $\epsilon>0$ on at least one bundle. The $k=1$ variation, with price adjustments $\bar{p}^{t+1}$ is thought to have better incentive properties (Wurman and Wellman 1999), and empirical analysis has demonstrated high efficiency with straightforward bidders (Wurman and Wellman 2000).

RAD provides an additive-or (OR) bidding language, and winner determination is formulated to allow multiple bids to be accepted from any one bidder (Kwasnica, Ledyard, Porter, and DeMartini 2004). Straightforward bidding is well defined for 
the OR language when valuations have additive-or semantics (e.g. when the bidder's value for a disjoint combination of packages is the sum of the individual package values). ${ }^{20}$ However, this OR language is not always expressive for straightforward bidding. For example, a bidder with valuation $(A B, \$ 20),(C D, \$ 20),(A B C D, \$ 20)$ facing prices $(A B, \$ 10)$ and $(C D, \$ 10)$ can not represent her best-response demand set (either $A B$ or $C D$ but not both) with an OR language.

RAD maintains linear and anonymous prices and formulates the price update as a series of LPs. The methodology is close in spirit to methods due to Rassenti, Smith and Bulfin (1982), where approximate prices are computed in a one-shot CA. ${ }^{21}$ Let $S^{t}=\left(S_{1}^{t}, \ldots, S_{n}^{t}\right)$ denote the provisional allocation computed in round $t$. RAD computes new linear prices that exactly match the bid price for all winning bids, with $\sum_{j \in S_{i}^{t}} p_{\text {ask }}^{t+1}(j)=p_{\mathrm{bid}, i}^{t}\left(S_{i}^{t}\right)$, and minimize the maximal regret across losing bids, with regret defined as the difference $\max \left\{0, p_{\text {bid }, i}^{t}(S)-\sum_{j \in S} p_{\text {ask }}^{t+1}(j)\right\}$. Ties are broken first to lexicographically lower the regret on as many losing bids as possible, and then on prices for items in winning bids to maximize the minimal price on each such bundle. This procedure ensures a unique solution and is designed to provide bidders with informative signals.

Experimental results in a laboratory with human bidders demonstrate that RAD achieves higher efficiency than non-combinatorial auctions (Banks, Ledyard, and Porter 1989). In addition, RAD is demonstrated to terminate with fewer rounds than the SAA design, which typically has fewer rounds than simple ascending-bid CAs (Cybernomics 2000).

Auctions $i$ BEA (Parkes and Ungar 2002) and MP (Mishra and Parkes 2004) are general purpose ascending Vickrey auctions. $i$ BEA extends $i$ Bundle(3) to adjust past the first set of CE prices and achieve UCE prices with straightforward bidding. This provides enough information to adjust downwards to VCG payments upon termination, bringing straightforward bidding into an ex post 
equilibrium for general values. Similarly, MP extends the minimal price update rule in dVSV, to ensure that the auction terminates with UCE prices. The same tradeoff occurs between $i \mathrm{BEA}$ and MP as occurs between $i$ Bundle and dVSV. Although one should expect MP to converge more quickly than $i \mathrm{BEA}$, each price update in $i \mathrm{BEA}$ is simple to compute and easier to explain to bidders.

\section{Non Price-Based Approaches}

We survey three examples of non price-based approaches to iterative CA design. These auctions do not require that bidders submit bids in response to ask prices. Instead, they include richer query models and are structured fundamentally different than ascending-price auctions. The auctions fall into one of the following categories:

Decentralized Approaches. The winner determination problem is moved to the bidders, who are responsible for submitting bids and also computing allocations of items with high revenue given existing bids. The Adaptive User Selection Mechanism (AUSM) (Banks, Ledyard, and Porter 1989), a continuous auction in which winner determination is distributed to bidders, provides a canonical example.

Proxy Auctions. Proxy agents, which automatically submit bids through a predetermined bidding procedure, provide an interface between bidders and an auction. Bidders provide incremental value information to proxy agents, which may query bidders actively.

Direct-Elicitation Approaches. (Conen and Sandholm 2001) Explicit queries are formulated by the auctioneer (perhaps in a decentralized way), and a bidder's strategy determines how to respond to these queries. Multi-party elicitation approaches are used to ensure that information reported by one bidder can be used to refine the queries asked of another bidder. 
There is perhaps some ambiguity between the proxy auctions approach and the direct-elicitation approach. We choose to reserve the term proxy auction to settings in which the proxy agents are restricted to following a straightforward bidding strategy in an auction protocol. Direct-elicitation methods may also distribute elicitation to individual proxy agents. However, the proxies in direct-elicitation interact with a richer centralized protocol (more akin to a computational procedure), that can itself be designed with knowledge that it will be interacting with automated proxy agents.

\subsection{Decentralized Approaches: The AUSM Design}

AUSM is a continuous auction that maintains a list of provisional winning bids and a standby queue. This standby queue contains bids that have been submitted but are not provisionally winning, and is designed to allow bidders to coordinate their bids. A bidder can always submit a bid to the queue and can also suggest a new combination of bids from the queue that provide more revenue than the current allocation. This proposed allocation becomes the new provisional allocation. The bidding language within the queue is implicitly additive-or and bidders are unable to place logical constraints between multiple bids in the queue. AUSM terminates after a period of quiescence.

AUSM distributes the winner-determination computation across the bidders. The auctioneer is only required to verify that a new provisional allocation is better than the current allocation and that it is formed from bids in the standby queue. Related ideas are found in the work of Brewer (1999) and the PAUSE auction (Land, Powell and Steinberg, Chapter 6).

On one hand, this decentralization can remove a computational bottleneck from iterative CAs. On the other hand, this decentralization can bias the outcome in favor of technologically sophisticated bidders better able to solve larger optimization problems. See Pekeč and Rothkopf (Chapter 16) and Parkes and Shneidman (2004) for an additional discussion of the incentive aspects of 
decentralized approaches to solving the winner-determination problem. Another potential concern with AUSM is that bidders must be able to process the disaggregated feedback provided in the auction, in the form of submitted bids.

Nevertheless, AUSM has been demonstrated to provide better allocative efficiency than a non-combinatorial auction in experiments with human bidders (Banks, Ledyard, and Porter 1989).

\subsection{Proxied Auctions}

Proxied auctions include automated proxy agents which interface between bidders and the auctioneer and submit bids following a predetermined procedure. In an ascending CA the proxies typically follow straightforward bidding strategies. If a proxy agent is following a first-best strategy (i.e. the bidding strategy that an agent would follow with full information about a bidder's value), then it must elicit enough information to compute a best-response to prices in each round. At one extreme, each proxy agent can require direct and complete revelation at the start of the auction (Ausubel and Milgrom 2002, also Chapter 3). Of course, this reduces the auction to a sealed-bid auction. However, when combined with a bidder-to-proxy interface that allows bidders to provide incremental value information, proxied auctions suggest a paradigm shift in iterative CAs from indirect revelation (e.g. via best-response bids to prices) to incremental but direct revelation (Parkes 2001, section 7.5).

Proxy agents can maintain partial information about valuations. For instance, this information could be in the form of exact values for a subset of bundles, or approximate values for each bundle. Proxy agents can decide when to query and when to bid, based on a model of costly elicitation.

The bidder-to-proxy interface need not be constrained to logical languages such as XOR or OR, and can be adapted to suit the local problem of a bidder. For example, a bidder in a logistics problem can define the constraints and costs for her local business problem. The ability to support this kind of expressiveness can 


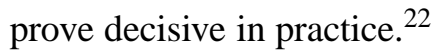

In addition to enriching the bidding language, proxy auctions can also offer the following advantages:

a) Proxy auctions can restrict the dynamic strategies available to bidders, for example by enforcing straightforward bidding based on reported valuations and by requiring consistent information-revelation to proxies (see Section 7.5, Parkes 2001, and Ausubel and Milgrom 2002).

b) Proxy auctions offer opportunities for accelerated implementations of auctions, because there can be multiple fast "proxy rounds" of bidding interleaved with a few "human rounds" to refine proxy's value information, see Hoffman, Menon, van der Heever, and Wilson (Chapter 17) and Wurman, Zhong and Cai (2004). ${ }^{23}$ In imposing strong activity rules, for instance to require that a bidder provides a consistent response to queries during an auction, one must allow for bidder mistakes and also for bidders that might be adjusting their beliefs about value as they receive feedback (e.g. in a correlated value setting). Ausubel, Cramton and Milgrom (Chapter 5) advocate using a relaxed consistency rule to provide incentives for early demand revelation while allowing for these other effects.

\subsection{Direct-Elicitation Approaches}

A direct-elicitation approach formulate queries about bidder valuations, to which bidders are expected to respond (although not necessarily truthfully). Queries are typically interleaved across bidders so that the queries asked of one bidder can be selected given responses by other bidders. In this way, complete elicitation can be avoided through focused elicitation on interesting parts of the allocation space.

Sandholm and Boutilier (Chapter 10) provide an extended discussion of direct-elicitation methods for the design of iterative CAs.

The query process in direct elicitation can be fully integrated within a winner-determination algorithm to determine whether enough information is available to implement an efficient allocation (Conen and Sandholm 2001, e.g.). 
The query process may also be defined through an algorithmic technique that does not have a very natural analogue with traditional auction designs, such as computational learning theory (Zinkevich, Blum, and Sandholm 2003, Lahaie and Parkes 2004a).

Example queries can include: "is bundle $S_{1}$ preferred to bundle $S_{2}$ ?"; "is your value on bundle $S_{1}$ at least $\$ 100$ ?"; and “what is your value on bundle $S_{1}$ ?.” The goal is to ask the minimal number of queries required to determine the efficient allocation and perhaps also to determine the VCG payments. Computing the VCG payments brings truthful response by bidders into an ex post equilibrium.

We know that any elicitation process must also determine CE prices if the goal is to determine an efficient allocation, and UCE prices if the VCG outcome is important (see Section 2). Thus, one reasonable approach is explicitly price based, with elicitation structured as a search for CE prices. One can also consider an allocation-based approach, with elicitation structured as a search for the efficient allocation.

Price based. Query bidders until the value information is sufficient to verify a set of UCE prices and a supporting allocation for the main economy. For instance, one can simulate learning algorithms to elicit bidder valuations until they are known with enough accuracy to determine UCE prices (Lahaie and Parkes 2004a, Lahaie and Parkes 2004b).

Allocation-based. Query bidders until the value information provides a certificate for the efficient allocation and the Vickrey payments. Use partial information to augment a search in allocation space, executing new queries to refine information that will resolve current uncertainty about the efficient allocation (Conen and Sandholm 2001, Hudson and Sandholm 2004).

As yet there are no published studies to compare the elicitation effectiveness and computational scalability of price-based approaches and allocation-based approaches. Price-based approaches may be fundamentally more scalable, with 
queries determined by solving optimization problems that are restricted by current bidder responses, for instance via winner-determination problems defined on bundles returned by best-response queries. In comparison, allocation-based approaches must strive to avoid maintaining an allocation graph that scales exponentially with the number of items. ${ }^{24}$

Price-based approaches are also naturally decentralized: in a proxied architecture, each proxy agent can elicit preference information independently until it has enough information to determine its best-response to current prices. This best-response information can verify that an allocation is efficient even though each proxy knows nothing about the values of other bidders.

Recently, methods from computational learning theory (CLT) have been adapted to direct elicitation. CLT provides membership queries ("what is your value on bundle $S$ ?") and equivalence queries ("is your valuation function $\hat{v}$ ? If not, identify a bundle $S$ for which $\hat{v}(S)$ is incorrect.") In one approach, each proxy is responsible for learning the exact value function of a single bidder in isolation (Zinkevich, Blum, and Sandholm 2003, Blum, Jackson, Sandholm, and Zinkevich 2004). In another approach, Lahaie and Parkes (2004a) integrate CLT into price-based approaches and use demand queries to simulate equivalence queries. A demand query presents prices $p$ and a bundle $S$ and asks whether $S$ is in the demand set of the bidder at the prices. This coordinates elicitation across proxy agents and provides an elicitation method that can terminate early as soon as CE prices are discovered and without learning values exactly.

\section{Summary}

Iterative CAs are of critical importance in addressing the problem of preference elicitation, which many view as the biggest issue to surmount in the real-world deployment of CAs. The sophisticated combinatorial optimization and pricing algorithms of CAs are impotent without rich bid information from bidders. Iterative CAs focus elicitation, often through price discovery, and can find 
efficient allocations without bidders reporting, or even computing, their exact value information. We emphasized price-based approaches, and in particular a primal-dual design paradigm. Canonical non-price based approaches, including proxied- and direct-elicitation approaches, were also discussed.

For a related discussion of the primal-dual approach to auction design see Chapter 8, and see also Chapters 3, 5 and 6 for discussions of specific iterative CAs. Chapters 9, 10 and 11 relate to the discussion of bidding languages, elicitation, and communication complexity. Chapter 17 discusses methods to accelerate the computation of the outcome of a proxied ascending price CA.

Looking ahead, we see a number of outstanding problems in the design of iterative CAs:

- Introduce the cost of preference elicitation more explicitly into the auction design problem. Current methods are mainly first best, and seek to find an efficient allocation with as little information as possible. But what happens when this minimal information remains too costly for bidders to provide? This is the problem of designing second-best auctions, that make the right tradeoff between the cost of information and the value of additional information in terms of improving the market allocation. Some initial progress has been made in the analysis of auction design with costly information (Compte and Jehiel 2000, Larson and Sandholm 2001, Fong 2003, Parkes 2004), and with bounded communication (Blumrosen and Nisan 2002, Blumrosen, Nisan, and Segal 2003), but much more work needs to be done.

- Design iterative CAs for which straightforward bidding is an ex post equilibrium, but which do not suffer from the well-known vulnerabilities of the VCG auction that are outlined by Ausubel and Milgrom (Chapter 1). These auctions will necessarily not be allocatively efficient, but may be more desirable due to new robustness against manipulation by coalitions and improved revenue properties. - Current auctions for general valuations for which theoretical results are available use XOR bidding languages which are not concise enough to be usable for many 
real-world applications. We need iterative CAs that support richer bidding

languages, for instance allowing side constraints, volume discounts, and other high-level bidding logic to be stated and then refined during the auction. 


\section{Notes}

${ }^{1}$ One argument commonly made for why very few VCG mechanisms are seen in practice is that bidders are reluctant to reveal their complete and true valuations in a situation of long-term strategic interaction (Rothkopf, Teisberg, and Kahn 1990).

${ }^{2}$ The observed vulnerabilities of the VCG auction can be viewed as problems intrinsic to the task of implementing efficient allocations in an ex post equilibrium in iterative CAs, given the uniqueness of the VCG auction among efficient auctions (see Chapter 1).

${ }^{3}$ Goods are substitutes is the largest set containing unit-demand valuations (with $v_{i}(S)=\max _{j \in S}\left\{v_{i j}\right\}$ for all $S$, where $v_{i j}$ is the value for item $j$ in isolation) for which the existence of linear CE prices can be established (Gul and Stacchetti 1999).

${ }^{4} \mathrm{Gul} \&$ Stacchetti (1999) show that there is often no linear price equilibrium that supports the VCG payments with substitutes valuations. On the other hand, linear prices can support the VCG outcome for unit-demand valuations (Leonard 1983).

${ }^{5}$ Computational analysis on a broad test suite of problem instances demonstrated failure of buyers are substitutes in around 43\% of instances (Parkes 2001, Chapter 7, pp.216).

${ }^{6}$ In fact, the prices will support all efficient allocations in each marginal economy because prices that support any one efficient allocation support all.

${ }^{7}$ Parkes (2002) uses agent-independence to refer to privacy-preservation. Parkes also requires an additional technical requirement (outcome-independence), that is without loss of generality for "best-response bidding languages," which are expressive enough to simulate at least the following bids: bundle $S_{1}$ is worth at least $\$ 100$; and bundle $S_{1}$ is worth at least $\$ 50$ more than bundle $S_{2}$; and bundle $S_{1}$ has value $\$ 200$.

${ }^{8}$ The form of activity rule used in the FCC spectrum auctions is due to Paul Milgrom and Robert Wilson. The rule requires quantities bid in the auction are (weak) monotonically decreasing. Similar rules have since become standard in ascending CAs.

${ }^{9}$ Roth and Ockenfels (2001) have studied the use of deadlines versus rolled closures, on eBay and Amazon Internet auctions respectively. Bidders on Amazon bid earlier than on eBay, and many bidders on eBay wait until the last seconds of 
an auction to bid.

${ }^{10}$ Click-box bidding was adopted by the FCC in the light of evidence that bidders used the trailing digits for signaling in early wireless spectrum auctions.

${ }^{11}$ Of course, arbitrary decommiting may be undesirable because it allows insincere bidding and cheap talk.

${ }^{12} \mathrm{BAS}$ holds and there is a set of minimal CE prices that will support the VCG outcome. However, Gul and Stacchetti's (2000) auction maintains item prices and a stronger condition, such as unit-demand valuations, is required for VCG payments to be supported with linear CE prices.

${ }^{13}$ A set of items, $S^{\prime} \subseteq \mathcal{G}$, are overdemanded when it is not possible to satisfy the demand sets of bidders that demand only items in $S^{\prime}$.

${ }^{14} \mathrm{~A}$ set $L \subseteq \mathcal{I}$ of bidders are undersupplied if not all bidders can be satisfied in the provisional allocation.

${ }^{15}$ One can also imagine that each round of the auction closes the duality gap between the feasible primal and dual solutions. At termination the duality gap is zero, complementary slackness holds, and we have an efficient allocation and CE prices.

${ }^{16}$ Recently, de Vries, Schummer and Vohra (2003) observe a formal distinction between the subgradient approach adopted in $i$ Bundle and the primal-dual approach adopted in dVSV and MP. One can view subgradient methods as a specialization of primal-dual, and thus we prefer to continue to adopt the primal-dual terminology throughout this section.

${ }^{17}$ Specifically, the bidder need only bid for bundles $S$ for which there are no bundles $S^{\prime} \subset S$ with $v_{i}\left(S^{\prime}\right)=v_{i}(S)$, i.e. taking advantage of sparse valuations.

${ }^{18} \mathrm{~A}$ simple way to achieve consistency is to use a proxy agent interface. The proxy can follow a straightforward bidding strategy based on value information reported by a bidder. A bidder can provide additional information as needed but must be consistent during the course of the auction.

${ }^{19}$ In particular, de Vries, Schummer, and Vohra (2003) note that $i$ Bundle is more correctly a subgradient algorithm while dVSV is a primal-dual algorithm. Primaldual algorithms are inherently faster than subgradient algorithms in the optimization literature (Fisher 1981).

${ }^{20}$ This property is satisfied by the "spatial fitting" environment used by Kwasnica, Ledyard, Porter and DeMartini (2004) in experiments and introduced in Banks, 
Ledyard and Porter (1989).

${ }^{21}$ Graves et al. (1993) have also described LP-based methods to provide price feedback in a multi-stage combinatorial auction procedure adopted at the University of Chicago Graduate Business School in the 1990's.

${ }^{22}$ For instance, Kalagnanam, Bichler, Davenport and Hohner (Chapter 23) and Caplice and Sheffi (Chapter 21) discuss the role of item prices coupled with volume discounts and complex bid-taker constraints in industrial procurement and logistics.

${ }^{23}$ Indeed, the speed of iterative combinatorial auctions has often been cited in FCC discussions as one potential drawback in comparison with linear price auctions.

${ }^{24}$ Current allocation-based algorithms cannot scale beyond a handful of bidders and tens of items (Hudson and Sandholm 2004). In comparison, ascendingprice auctions readily scale to problems that push the limit of current winnerdetermination technology (Parkes and Ungar 2000a). We are not aware of any computational studies of price-based direct elicitation methods such as those of Lahaie and Parkes (2004a).

\section{Appendix: LP Theory}

Consider the linear program:

$$
\begin{array}{r}
\max c^{T} x \\
\text { s.t. } \quad A x \leq b \\
x \geq 0
\end{array}
$$

where $A$ is a $m \times n$ integer matrix, $x \in R^{n}$ is a $n$-vector, and $c$ and $b$ are $n-$ and $m$-vectors of integers. Vectors are column-vectors, and notation $c^{T}$ indicates the transpose of vector $c$, similarly for matrices. The primal problem [P] is to compute a feasible solution for $x$ that maximizes the value of the objective function. 
The dual program is constructed as:

$$
\begin{array}{r}
\min b^{T} y \\
\text { s.t. } \quad A^{T} y \geq c \\
y \geq 0
\end{array}
$$

where $y \in R^{m}$ is a $m$-vector. The dual problem is to compute a feasible solution for $y$ that minimizes the value of the objective function.

Let $V_{\mathrm{LP}}(x)=c^{T} x$, the value of feasible primal solution $x$, and $V_{\mathrm{DLP}}(y)=b^{T} y$, the value of feasible dual solution $y$.

Complementary-slackness conditions express logical relationships between the values of primal and dual solutions that are necessary and sufficient for optimality.

\section{Definition 6 (Complementary-Slackness). Complementary-slackness} conditions constrain pairs of primal and dual solutions. Primal CS conditions state $x^{T}\left(A^{T} y-c\right)=0$, or in logical form:

$$
x_{j}>0 \Rightarrow A^{j} y=c_{j}
$$

where $A^{j}$ denotes the jth column of $A$ (written as a row vector to avoid the use of transpose). Dual CS conditions state $y^{T}(A x-b)=0$, or in logical form:

$$
y_{j}>0 \Rightarrow A_{i} x=b_{i}
$$

where $A_{i}$ denotes the $i$ th row of $A$.

Theorem 10 (strong-duality). A pair of feasible primal, $x$, and dual solutions, $y$, are primal and dual optimal if and only if they satisfy the complementary-slackness conditions.

Proof. Primal CS holds iff $x^{T}\left(A^{T} y-c\right)=0$, and Dual CS holds iff $y^{T}(A x-b)=0$. Equating, and observing that $x^{T} A^{T} y=y^{T} A x$, we have P-CS and D-CS iff $x^{T} c=y^{T} b$, or $c^{T} x=b^{T} y$. The LHS is the value of the primal, $V_{\mathrm{LP}}(x)$, and the RHS is the value of the dual, $V_{\mathrm{DLP}}(y)$. By the strong duality 
theorem, $V_{\mathrm{LP}}(x)=V_{\mathrm{DLP}}(y)$ is a necessary and sufficient condition for the solutions to be optimal.

\section{References}

Ausubel, Lawrence M, 2002, “An Efficient Dynamic Auction for Heterogeneous Commodities," Discussion paper, Department of Economics, University of Maryland.

Ausubel, Lawrence M, and Paul Milgrom, 2002, “Ascending Auctions with Package Bidding," Frontiers of Theoretical Economics, 1, 1-42.

Banks, Jeffrey S, John O Ledyard, and David Porter, 1989, “Allocating Uncertain and Unresponsive Resources: An Experimental Approach," The Rand Journal of Economics, 20, 1-25.

Bertsekas, Dimitri P, 1987, Dynamic Programming: Deterministic and Stochastic Models, Prentice-Hall.

Bikhchandani, Sushil, and Joseph M Ostroy, 2002, "The Package Assignment Model," Journal of Economic Theory, 107, 377-406.

Blum, Avrim, Jeffrey Jackson, Tuomas Sandholm, and Martin Zinkevich, 2004, "Preference Elicitation and Query Learning," Journal of Machine Learning Research (JMLR), 5, 649-667.

Blumrosen, Liad, and Noam Nisan, 2002, "Auctions with severely bounded communication," in Proc. 43rd Annual Symposium on Foundations of Computer Science (FOCS 2002), pp. 406-415.

Blumrosen, Liad, Noam Nisan, and Ilya Segal, 2003, "Multi-Player and Multi-Round Auctions with Severely Bounded Communication," in Proc. 11th Annual European Symposium on Algorithms (ESA 2003), pp. 102-113. 
Brewer, Paul J, 1999, "Decentralized Computation Procurement and

Computational Robustness in a Smart Market," Economic Theory, 13, 41-92.

Compte, Olivier, and Philippe Jehiel, 2000, "On the virtues of the ascending price auction: New insights in the private value setting," Discussion paper, CERAS and UCL.

Conen, Wolfram, and Tuomas Sandholm, 2001, "Preference Elicitation in Combinatorial Auctions," in Proc. 3rd ACM Conf. on Electronic Commerce (EC-01), pp. 256-259. ACM Press, New York.

Cybernomics, 2000, "An experimental comparison of the Simulataneous Multiple Round Auction and the CRA Combinatorial Auction," Discussion paper, Report to the Federal Communications Commission, Available at http://wireless.fcc.gov/auctions/conferences/combin2000/releases/98540191.pdf.

de Vries, Sven, James Schummer, and Rakesh V Vohra, 2003, “On Ascending Vickrey Auctions for Heterogeneous Objects," Discussion paper, MEDS, Kellogg School, Northwestern University.

Demange, Gabrielle, David Gale, and Marilda Sotomayor, 1986, "Multi-item auctions," Journal of Political Economy, 94, 863-872.

Fisher, Marshall L, 1981, “The Lagrangian relaxation method for solving integer programming problems," Management Science, 27, 1-18.

Fong, Kyna, 2003, "Multi-stage Information Acquisition in Auction Design," Undergraduate thesis, Harvard University. Available at http://www.eecs.harvard.edu/econcs.

Graves, Robert L, Linus Schrage, and Jayaram Sankaran, 1993, “An Auction Method for Course Registration," Interfaces, 23, 81-92.

Gul, Faruk, and Ennio Stacchetti, 1999, "Walrasian equilibrium with Gross Substitutes," Journal of Economic Theory, pp. 95-124. 
Gul, Faruk, and Ennio Stacchetti, 2000, “The English Auction with Differentiated Commodities," Journal of Economic Theory, pp. 66-95.

Hoffman, Karla, 2001, "Issues in Scaling Up the 700 MHz Auction Design," Available at http://wireless.fcc.gov/auctions/conferences/combin2001/papers/Wye_river_DAC.pdf.

Hudson, Benoît, and Tuomas Sandholm, 2004, "Effectiveness of Query Types and Policies for Preference Elicitation in Combinatorial Auctions," in Proc. 3rd Int. Joint. Conf. on Autonomous Agents and Multi Agent Systems, pp. 386-393.

Kelso, Alexander S, and Vincent P Crawford, 1982, "Job matching, coalition formation, and gross substitutes," Econometrica, 50, 1483-1504.

Kwasnica, Anthony M, John O Ledyard, David Porter, and Christine DeMartini, 2004, “A New and Improved Design for Multi-object Iterative Auctions," Management Science, To appear.

Lahaie, Sébastien M, and David C Parkes, 2004a, “Applying Learning Algorithms to Preference Elicitation," in Proc. 5th ACM Conf. on Electronic Commerce, pp. $180-188$.

Lahaie, Sébastien M, and David C Parkes, 2004b, “Applying Learning Algorithms to Preference Elicitation in the Generalized Vickrey Auction," Discussion paper, Harvard University.

Larson, Kate, and Tuomas W Sandholm, 2001, "Costly Valuation Computation in Auctions," in Proc. Theoretical Aspects of Rationality and Knowledge VII, pp. 169-182.

Lehmann, Benny, Daniel Lehmann, and Noam Nisan, 2001, “Combinatorial Auctions with Decreasing Marginal Utilities," in Proc. 3rd ACM Conference on Electronic Commerce, pp. 18-28. ACM Press, To appear, Games and Economic Behavior. 
Leonard, Herman B, 1983, "Elicitation of honest preferences for the assignment of individuals to positions," Journal of Political Economy, 91, 461-479.

Milgrom, Paul, 2000, "Putting Auction Theory to Work: The Simultaneous Ascending Auction," Journal of Political Economy, 108, 245-272.

Milgrom, Paul, and Robert J Weber, 1982, “A theory of auctions and competitive bidding," Econometrica, 50, 1089-1122.

Mishra, Debasis, and David Parkes, 2004, “Ascending Price Vickrey Auctions using Primal-Dual Algorithms," Discussion paper, Harvard Univeristy.

Nisan, Noam, and Ilya Segal, 2003, "The Communication Requirements of Efficient Allocations and Supporting Lindahl Prices," Discussion paper, Hebrew University and Stanford University.

Papadimitriou, Christos H, and Kenneth Steiglitz, 1998, Combinatorial Optimization: Algorithms and Complexity, Dover.

Parkes, David C, 2001, "Iterative Combinatorial Auctions: Achieving Economic and Computational Efficiency," Ph.D. thesis, Department of Computer and Information Science, University of Pennsylvania, http://www.eecs.harvard.edu/parkes/diss.html.

Parkes, David C., 2002, "Price-Based Information Certificates for Minimal-Revelation Combinatorial Auctions," in Agent Mediated Electronic Commerce IV: Designing Mechanisms and Systems, vol. 2531 of Lecture Notes in Artificial Intelligence, pp. 103-122.

Parkes, David C., 2004, “Auction Design with Costly Preference Elicitation,” Annals of Mathematics and AI, To appear.

Parkes, David C., and Jeffrey Shneidman, 2004, "Distributed Implementations of Vickrey-Clarke-Groves Mechanisms," in Proc. 3rd Int. Joint Conf. on Autonomous Agents and Multi Agent Systems, pp. 261-268. 
Parkes, David C, and Lyle H Ungar, 2000a, "Iterative Combinatorial Auctions:

Theory and Practice," in Proc. 17th National Conference on Artificial Intelligence (AAAI-00), pp. 74-81.

Parkes, David C, and Lyle H Ungar, 2000b, "Preventing Strategic Manipulation in Iterative Auctions: Proxy Agents and Price-Adjustment," in Proc. 17th National Conference on Artificial Intelligence (AAAI-00), pp. 82-89.

Parkes, David C, and Lyle H Ungar, 2002, “An Ascending-Price Generalized Vickrey Auction,” Discussion paper, Harvard University, Presented at the SITE Workshop on The Economics of the Internet, Stanford University.

Porter, David, Steve Rassenti, and Vernon Smith, 2003, “Combinatorial Auction Design," Proc. of the National Acadamy of Sciences, 100, 11153-11157.

Rassenti, Steve J, Vernon L Smith, and R L Bulfin, 1982, “A combinatorial mechanism for airport time slot allocation,” Bell Journal of Economics, 13, $402-417$.

Roth, Alvin E, and Axel Ockenfels, 2001, "Last-Minute Bidding and the Rules for Ending Second-Price Auctions: Evidence from eBay and Amazon Auctions on the Internet," American Economic Review, forthcoming.

Rothkopf, Michael H, T J Teisberg, and E P Kahn, 1990, "Why are Vickrey Auctions Rare?," Journal of Political Economy, 98, 94-109.

Wired, 2000, "Capitalist Econstruction," Wired magazine, By Chip Bayers, and available at http://www.wrsasc.com/press/pressCapitalistEconstruction.pdf.

Wurman, Peter R, and Michael P Wellman, 1999, "Equilibrium Prices in Bundle Auctions," in Proc. AAAI-99 Workshop on Artificial Intelligence for Electronic Commerce, pp. 56-61. 
Wurman, Peter R, and Michael P Wellman, 2000, “AkBA: A Progressive, Anonymous-Price Combinatorial Auction," in Second ACM Conference on Electronic Commerce, pp. 21-29.

Wurman, Peter R, Jie Zhong, and Gangshu Cai, 2004, "Computing price trajectories in combinatorial auctions with proxy bidding," Electronic Commerce Research and Applications, To Appear.

Zinkevich, Martin, Avrim Blum, and Tuomas Sandholm, 2003, "On polynomial-time preference elicitation with value queries," in Proc. 4th ACM Conf. on Electronic Commerce, pp. 176-185. 


\begin{tabular}{|l|ccc|}
\hline \hline & A & B & AB \\
\hline Bidder 1 & $30^{*}$ & 0 & 30 \\
Bidder 2 & 0 & $40^{*}$ & 40 \\
Bidder 3 & 0 & 20 & 40 \\
Bidder 4 & 25 & 0 & 25 \\
Bidder 5 & 0 & 25 & 25 \\
\hline \hline
\end{tabular}

\begin{tabular}{|c|}
\hline \hline minimal information set \\
\hline$v_{1}(A) \geq v_{1}(B), v_{1}(A) \geq v_{1}(A B), v_{1}(A) \geq 25$ \\
$v_{2}(B) \geq v_{2}(A), v_{2}(B) \geq v_{2}(A B), v_{2}(B) \geq 25$ \\
$v_{3}(A) \leq 0, v_{3}(B) \leq 20, v_{3}(A B) \leq 40$ \\
$v_{4}(A B) \leq 25$ \\
$v_{5}(A B) \leq 25$ \\
\hline \hline
\end{tabular}

(a)

(b)

Figure 2.1: Example 2.2: (a) Bidder valuations, with the efficient allocation indicated by *. (b) Minimal information on bidder valuations to compute the VCG outcome.

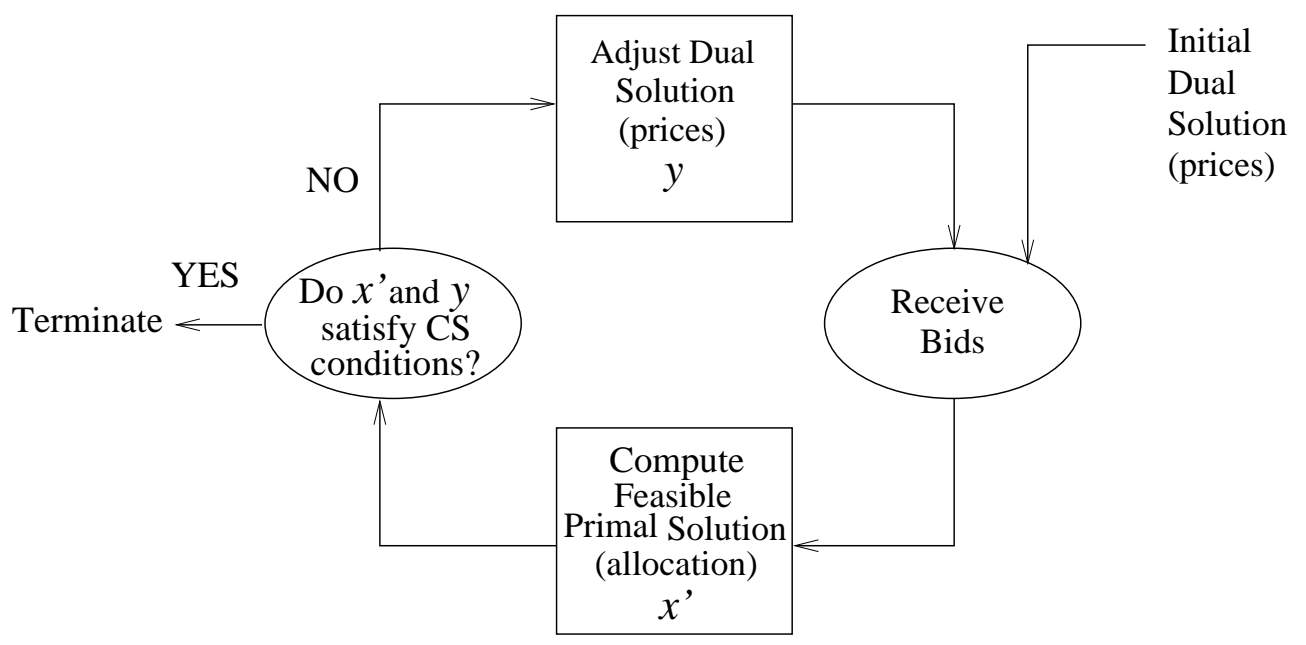

Figure 2.2: A Primal-Dual Interpretation of an Ascending CA.

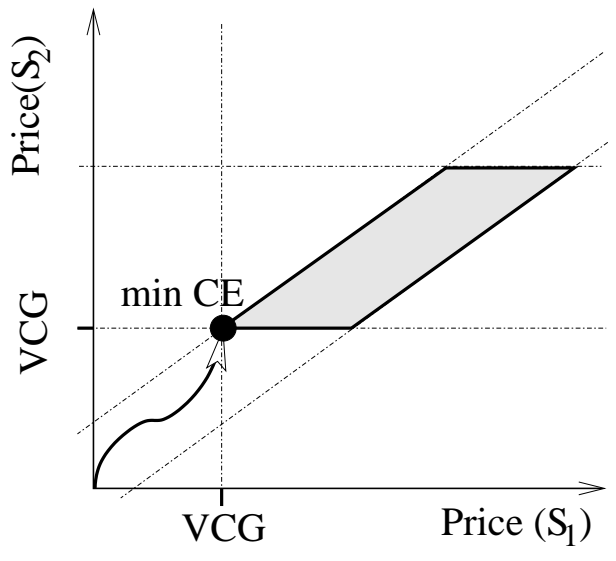

(a) BSM holds.

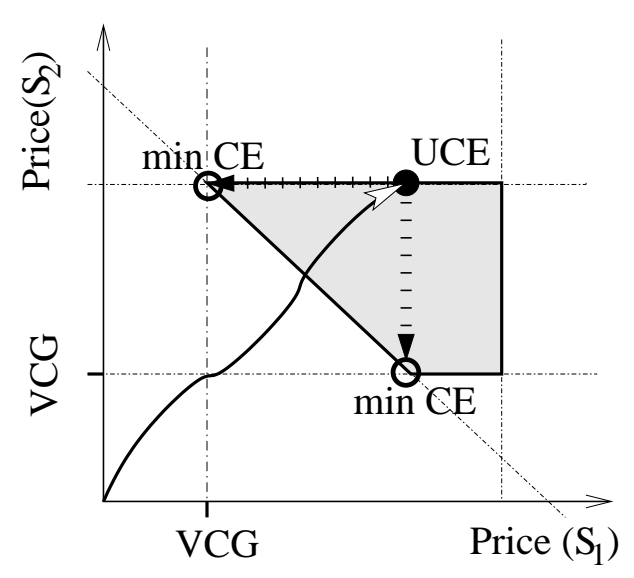

(b) BAS fails.

Figure 2.3: Adjusting towards the VCG outcome in price-based iterative CAs.

CE prices lie within the shaded regions. 